\title{
KAHBPS-2
}

\section{Epidemiology and future prediction of Korean liver, pancreatic and biliary cancer}

\author{
Sung-Sik HAN* \\ Department of Surgery, National Cancer Center, Goyang, Korea
}

Lecture: Supported by a national policy on cancer prevention and screening in Korea, the incidence of all types of cancers peaked in 2011 and turned into a declining trend. The overall cancer mortality rate has also steadily decreased since 2002 due to advances in diagnostic and treatment modalities. In 2018, liver, pancreas, gallbladder and biliary tract cancer were the 6th (6.5\% of all cancers), 8th (3.1\%), and 9th (2.9\%) most common cancers in Korea [1]. Although the incidence of hepatocellular carcinoma is decreasing due to vaccination and effective treatment for viral hepatitis, incidences of pancreatic and biliary tract cancer are rapidly increasing. Moreover, liver and pancreatic cancer ranked the 2nd and 5th most common causes of death in 2019 [2]. However, due to relatively small number of patients, liver, pancreatic and biliary tract cancer have not been given a priority in national health policy, despite being fatal. Moreover, there are no studies related to future predictions regarding long-term changes in incidence and mortality. Therefore, the purpose of this study was to predict epidemiologic features of liver, pancreatic, and biliary tract cancer to build evidence that could provide insight into health policy and budget allocation, including cancer prevention, diagnosis and treatment strategies.

\section{Incidence and mortality rate of liver, pancreas, and biliary tract cancer in Korea from 1999 to 2017}

Summary statistics of liver cancer (C22) was retrieved from annual report of cancer statistics published by Korea Central Cancer Registry [1]. For pancreatic and biliary tract cancer, incidence data was retrieved from Korea Central Cancer Registry, National Cancer Center. Individuals with corresponding International Statistical Classification of Diseases and Related Health Problems (ICD)10 codes and international classification of diseases for oncology (ICD-O) morphology codes were categorized as follows; gallbladder cancer (C23), intrahepatic bile duct cancer (C22.1, excluding M8162/3), extrahepatic bile duct cancer (C24.0 and M8162/3), ampulla of Vater cancer (C24.1), and pancreatic cancer (C25).

Individuals with a morphology code "M8162/3" were reclassified as extrahepatic bile duct cancer because changes in topographic classification of Klatskin tumor overestimated cases of intrahepatic bile duct cancer [3]. However, mortality data retrieved from Statistics Korea from 2002 to 2018 did not have an identifier to reclassify the bile duct cancer accordingly. Therefore, mortality rate analysis and future epidemiology prediction utilized the sum of intra- and extrahepatic bile duct cancer as a single category for consistent analysis. From 2009 to 2018, annual percentage change (APC) of age-standardized incidence rate (ASIR, per 100,000 population) of liver cancer was $-4.1 \%$ (24.0 to 16.7) [1]. From 1999 to 2017 trends in ASIR of each cancer were as follows; 2.9 to 2.6 (gallbladder [GB], APC -1.03\%, $p<0.0001$ ), 2.0 to 2.7 (intrahepatic bile duct [IBD], APC 2.30\%, $p=0.0033$ ), 2.9 to 3.2 (extrahepatic bile duct [EBD], APC 0.93\%, $p=$ 0.0009 ), 0.9 to 0.9 (ampulla of Vater [AoV], APC $-0.37 \%, p=0.1992$ ), and 5.6 to 7.1 (pancreas, APC $1.46 \%, p<0.0001$ ). Especially, APC of pancreatic cancer ASIR in females was significantly higher than in males ( $2.30 \%$ vs. $0.61 \%, p<0.0001)$. Male to female ASR ratio decreased in GB (1.08 to 1.00), IBD (2.55 to 2.18), EBD (2.28 to 1.94), and pancreatic cancer (1.95 to 1.45 ).

Age-standardized mortality rate (ASMR) of each cancer was as follows; 18.7 to 8.0 (liver), 2.4 to 1.6 (GB), 5.5 to 4.6 (IBD and EBD), 0.4 to $0.4(\mathrm{AoV})$ and 5.5 to 5.6 (pancreas).

\section{Predicting future epidemiology of liver, pancreatic, and biliary tract cancer in Korea until 2040}

Incidence and mortality rate was predicted using an age-period-cohort model $(\lambda$ (age, period) $=g\{f A($ age $)+f P($ period $)+f C$ (cohort) $\} ; \lambda$, incidence [mortality] rate as a function of age and calendar period; $g$, 'link' function; $f A$, function of age; $f P$, function 
of period [year of incidence or mortality]; $f C$, function of cohort [year of birth, i.e., cohort=period-age]) [4]. Due to inconsistent coding practice in the causes of death statistics, ICD-10 codes used for prediction on future incidence and mortality were categorized as follows; Liver cancer (C22.0, C22.9), GB cancer (C23), IBD and EBD cancer (C22.1, C24.0, C24.8, C24.9), AoV cancer (C24.1), and pancreatic cancer (C25). To adjust future demographic structure, estimated population by Statistics Korea was applied to calculate ASIRs and ASMRs.

Predicted annual cases of newly diagnosed cancer from 2021 to 2040 increased in all types of cancers as follows; 12,169 to 13,089 (liver), 2,857 to 4,038 (GB), 6,600 to 7,964 (IBD and EBD), 935 to 1,376 (AoV), 8,578 to 16,170 (pancreas). Predicted ASIR increased only in pancreatic cancer (7.5 to 8.2), while other cancers had declining trends; 12.0 to 8.5 (liver), 2.3 to 1.7 (GB), 5.4 to 3.2 (IBD and EBD), and 0.8 to $0.6(\mathrm{AoV})$.

Predicted annual deaths from 2021 to 2040 decreased in liver cancer (7,119 to 6,037), while other cancers had increasing trends as follows; 1,822 to 2,391 (GB), 5,598 to 7,928 (IBD and EBD), 408 to 536 (AoV), and 6,598 to 11,023 (pancreas). Predicted ASMR decreased in all types of cancers; 6.5 to 3.2 (liver), 1.4 to 0.9 (GB), 4.3 to 2.9 (IBD and EBD), 0.3 to 0.2 (AoV), and 5.4 to 4.7 (pancreas).

\section{Conclusion}

Pancreatic cancer revealed an increasing trend in ASIR, and the projected cases in 2040 are expected to outnumber those of liver cancer where ASIR is rapidly declining. ASIRs of GB, IBD and EBD, and AoV cancer had declining trends. Predicted ASMRs tended to decrease in all types of cancers, and ASMR of pancreatic cancer surpasses that of liver cancer from 2026.

Epidemiologic analysis and prediction should be conducted on an ongoing bases by researchers, including clinicians. Based on the accumulated results, we look forward to the establishment and implementation of a better national cancer policy in the future.

\section{References}

1. Korea Central Cancer Registry. Annual report of cancer statistics in Korea in 2018 [Internet]. Goyang: Korea Central Cancer Registry. Available from: https://ncc.re.kr/cancerStatsView.ncc?bbsnum=558\&searchKey=total\&searchValue=\&pageNum=1.

2. Statistics Korea. Causes of Death Statistics in 2019 [Internet]. Daejeon: Statistics Korea. Available from: http://kostat.go.kr/portal/ eng/pressReleases/8/10/index.board?bmode $=$ read $\& b S e q=\& a S e q=385629 \&$ pageNo $=1 \&$ rowNum $=10 \&$ navCount $=10 \& c u r r P g=\&-$ searchInfo=\&sTarget $=$ title\&sTxt $=$.

3. Kang MJ, Lim J, Han SS, Park HM, Kim SW, Won YJ, et al. Impact of changes in the topographic classification of Klatskin tumor on incidence of intra- and extrahepatic bile duct cancer: A population-based national cancer registry study. J Hepatobiliary Pancreat Sci 2021. https://doi.org/10.1002/jhbp.916 [in press]

4. Rosenberg PS, Check DP, Anderson WF. A web tool for age-period-cohort analysis of cancer incidence and mortality rates. Cancer Epidemiol Biomarkers Prev 2014;23:2296-2302. 